\title{
Accuracy improvement of avalanche photodiode based filter-less spectroscopy by deep learning
}

\author{
Masahiro Akiyama $^{{ }^{*}}$, Takaya Ishikawa ${ }^{2}$, Takashi Miyazaki ${ }^{1}$ \\ ${ }^{1}$ NIT Nagano College, 716 Tokuma Nagano-shi, Nagano 381-8550, Japan \\ ${ }^{2}$ Chiba University, 1-33 Yayoi-cho Inage-ku Chiba-shi, Chiba 263-8522, Japan \\ *akiyama@nagano-nct.ac.jp
}

\section{Abstract}

In this paper, we report a highly sensitive filter-less spectroscopy method using an avalanche photodiode (APD) by deep learning. Many different band pass filters or grating methods are commonly employed. Electron multiplying charge-coupled devices (CCD) are commonly used for high sensitivity sensing. However, conventional devices consisting of filters or gratings provide a relatively huge system size. In addition, the light intensity diminishes at the filters/gratings. Previously, experimental measurements using the proposed APD-based spectroscopy method exhibits the sensing capability to detect and divide dual wavelength light without band pass filters or grating for applying fluorescence and excitation lights detection. However, the proposed spectroscopy method has a problem in calculation with noises. The noises lead an inaccurate answer. So we propose a new method with deep learning instead of the calculation for improving spectroscopy accuracy.

Keywords: Avalanche photodiode, Filter-less, Deeplearning

\section{Introduction}

Conventionally, fluorescent labels and an expensive fluorescent scanner are used for DNA analysis [1]. The fluorescent scanner requires a special light source with a band pass filter [2]. It is, in consequence, relatively bulky by the filters. On the other hand, the biochemistry field has need of a straightforward space-saving fluorescence detection system with filter-less $[3,4]$. The filter-less systems are used characteristics of absorption coefficients $(\alpha)$ for sensing multi-wavelength fluorescence light.

Previously, we reported the APD-based filter-less spectroscopy method by complex calculation [5]. The principle of this APD-based highly sensitive spectroscopy method is used the varying multiplication ratio that is decided by incident light wavelength. The wavelength controls running distance of photo-excited carrier by absorption coefficients $(\alpha)$, and this element decides multiplication ratio on fixed high electrical field. So we can easily notice the wavelength of single wavelength light. In this case, we use essential formula as follow,

$$
M_{M I X V}=X_{\lambda_{1}} M_{\lambda_{1} V}+X_{\lambda_{2}} M_{\lambda_{2} V}+\cdots+X_{\lambda n} M_{\lambda_{n} V}
$$

$M_{M I X V}$ is a multiplication ratio at applying $V$ voltage and the lighting several wavelengths (mixed light). $M_{\lambda_{n} V}$ is a multiplication ratio at applying $V$ voltage and a lighting wavelength is $\lambda n[\mathrm{~nm}] . X_{\lambda n}$ is photocurrent ratio by the $\lambda n$ wavelength light. We can establish formulas in each apply voltages as follows,

$$
\begin{gathered}
M_{M I X V_{1}}=X_{\lambda_{1}} M_{\lambda_{1} V_{1}}+X_{\lambda_{2}} M_{\lambda_{2} V_{1}}+\cdots+X_{\lambda_{n}} M_{\lambda_{n} V_{n 1}} \\
M_{M I X V_{2}}=X_{\lambda_{1}} M_{\lambda_{1} V_{2}}+X_{\lambda_{2}} M_{\lambda_{2} V_{2}}+\cdots+X_{\lambda_{n}} M_{\lambda_{n} V_{n 2}} \\
\vdots \\
M_{M I X V_{n}}=X_{\lambda_{1}} M_{\lambda_{1} V_{n}}+X_{\lambda_{2}} M_{\lambda_{2} V_{n}}+\cdots+X_{\lambda_{n}} M_{\lambda_{n} V_{n}}
\end{gathered}
$$

$M_{M I X V n}$ is multiplication ratio at applying $V n$ voltage and lighting several wavelengths (mixed light). $M_{\lambda_{n} V n}$ is multiplication ratio at applying $V n$ voltage and lighting the $\lambda n[\mathrm{~nm}]$ wavelength light. From these equations, we can get $X_{\lambda_{1}} \ldots X_{\lambda_{n}}$ values by calculation as follows,

$$
\left[\begin{array}{c}
X_{\lambda_{1}} \\
X_{\lambda_{2}} \\
\vdots \\
X_{\lambda_{n}}
\end{array}\right]=\left[\begin{array}{cccc}
M_{\lambda_{1} V_{1}} & M_{\lambda_{2} V_{1}} & \cdots & M_{\lambda_{n} V_{n 1}} \\
M_{\lambda_{1} V_{2}} & M_{\lambda_{2} V_{2}} & \cdots & M_{\lambda_{n} V_{2}} \\
\vdots & \vdots & \ddots & \vdots \\
M_{\lambda_{1} V_{n}} & M_{\lambda_{2} V_{n}} & \cdots & M_{\lambda_{n} V_{n}}
\end{array}\right]^{-1}\left[\begin{array}{c}
M_{M I X V_{1}} \\
M_{M I X V_{2}} \\
\vdots \\
M_{M I X V_{n}}
\end{array}\right]
$$

However, the complex equations are not satisfied when the $M_{\lambda_{n V n}}$ have a noise. There are several noises, in this 
measurement system, that are shot noise of photocurrent, dark current noise, excess noise. So we change the calculation method to the deep learning for improving accuracy.

In this report, we divide dual wavelengths light that are blue and green lights for evaluating the accuracy. Our goal is classification at a noise level signal.

\section{Experimental details}

Figure 1 shows the measurement system. These incident lights (blue and green lights) have different wavelengths. So an APD makes different multiplication ratio. Thus this method can separate two types of light by using multiplication ratios of APD without band pass filters/gratings. In this measurement circuit, we employ APD (S6045-6), voltage source, and Pico ampere current meter (4200scs).

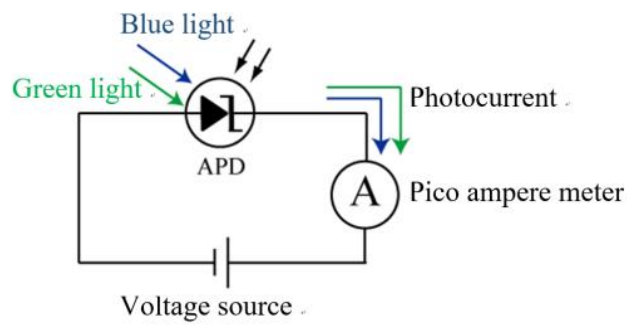

Fig. 1. Measurement circuit for photocurrent and dark current.

Firstly, the system measures dark current $(I d)$, and mixed photocurrent $(I p)$ characteristics for applied voltages in $1 \mathrm{~V}$ steps (0 to $210 \mathrm{~V}$ ) by Pico ampere current meter. The photocurrent ratio of blue and green light is changed 6 types that are 1:1, $1: 0.1,1: 0.01,1: 0.001$ and 1:0.0001. Secondly, the mixed multiplication $\operatorname{ratio}(M)$ is calculated by these currents $(I d, I p$, $I p O)$. The $I p O$ is photocurrent value when at applied $30 \mathrm{~V}$. The I $p$ and multiplication ratios are calculated by next equations,

$$
\begin{gathered}
I_{p}=I-I_{d} \\
M=\frac{I_{p}}{I_{p 0}}
\end{gathered}
$$

Figure 2 shows Ip characteristics for applied voltage at each photocurrent ratios. The $I p 0$ is around 1.3 and $0.6 \mu \mathrm{A}$ when the ratio is $1: 1$ and 1:0.0001 respectively. The photocurrent values of "ratio 1" change $1-6 \mu \mathrm{A}$ with random for making deep learning dataset. The $I p$ increase rapidly from 150 to $211 \mathrm{~V}$. Figure 3 shows multiplication ratios for applied voltage at each photocurrent ratios.

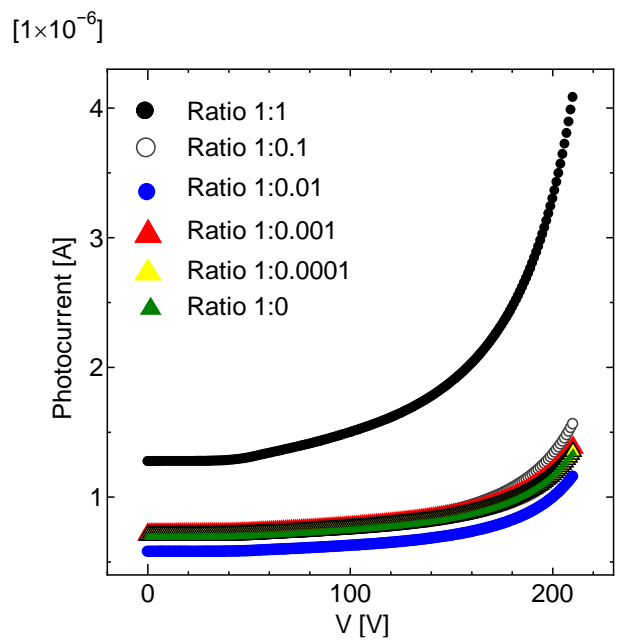

Fig. 2. Mixed photocurrent - voltage characteristics at each photocurrent ratios.

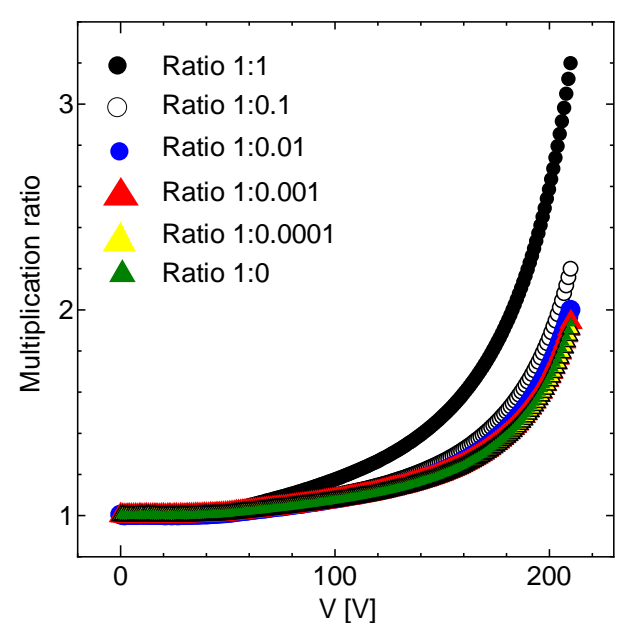

Fig. 3. Multiplication ratios at each photocurrent ratios.

The multiplication ratios are increased from $30 \mathrm{~V}$. The $M$ data are clearly different at the ratio 1:1, 1:0.1. However, the $M$ data are difficult to find different points at the ratio 1:0.01, 1:0.001, 1:0.0001 and 1:0. Figure 4 shows the dark current for apply voltage 6 times.

$\left[1 \times 10^{-8}\right.$ ]

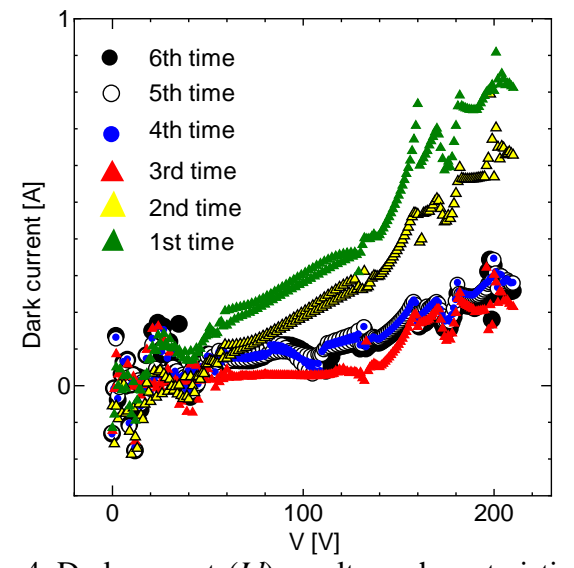

Fig. 4. Dark currents $(I d)$ - voltage characteristics. 
The dark currents are different by dark current generation process. The dark currents have fluctuation noise that the noise value is 4 to $7 \mathrm{nA}$. The dark current consist of 4 types. They are diffusion current by minority carrier, generation current from crystal fault, surface leakage current, tunnel current. The carrier generation timing and place that are not the same. It makes fluctuation noise of the dark current. The dark current remove from the value. However, the fluctuation noise is existing in the photocurrent. In the other hand, the photocurrent of green is below $6 \mathrm{nA}$ when the ratio is below 1:0.01 at 30V. So the fluctuation noise is almost same to the photocurrent of green when the photocurrent ratio at 1:0.01. It is indicated that the lights dividing is so difficult because the noise is almost same or bigger than green light signal when the photocurrent ratios are 1:0.01, 1:0.001, 1:0.0001 and 1:0. Our goal set classification $\operatorname{Ratio}(1: 0.01)$ that the signal value is almost the same as fluctuation noise. If we achieve this goal it indicates this method can divide incident lights when there is a high noise. Next chapter, we try light classifying by deep learning at the ratios are 1:0.01, 1:0.001, 1:0.0001 and 1:0.

\section{Data for deep learning}

We measured the 6 types of photocurrent ratio 960 times each. So the total number of measurement times of photocurrent-voltage characteristic is 5760. 211 data of photocurrent value from I-V result is able to be obtained, so the total amount of data is $5760 \times 211$. The data of deep learning has two types that are learning data and evaluation data. And learning data has two types that are practicing data and testing data. We set the dataset ratio that evaluating data ratio is $25 \%$ (1440), the practicing data ratio is $60 \%$ (3456) and testing data ratio is $15 \%$ (864). Figure 5 shows each percentage of the dataset for using deep learning. The following two types of data, the learning data, and the evaluation data, are required for analysis using deep learning.

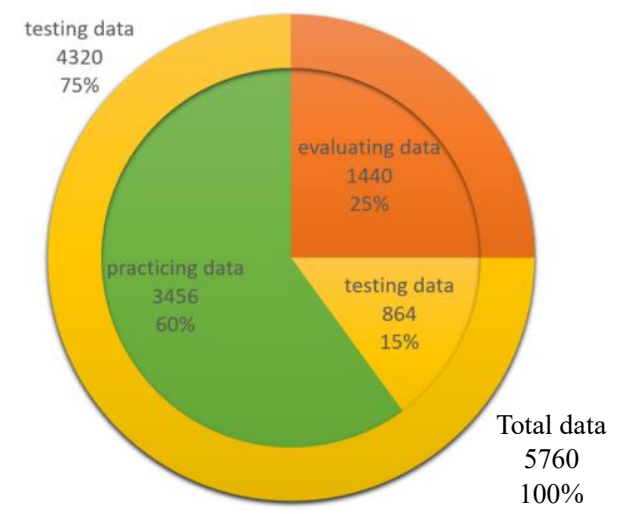

Fig. 5. Percentage of the dataset for using deep learning.

\section{Preprocess}

In this deep learning, we use three types of preprocessing that are multiplication ratio $(M)$ and the 1 st derivative multiplication ratio $\left(\frac{d M}{d v}\right)$ and the 2nd derivative multiplication ratio $\left(\frac{d^{2} M}{d v^{2}}\right)$. The photocurrent data has two types of information that are total incident light power and the wavelength. However, the total incident light power is not necessary for classifying. So the photocurrent data are converted to multiplication ratio $(M)$ with equation 5 , the $M$ data do not have the incident light power information, for highlighting the wavelength information. In addition, the $M$ data is converted to the 1 st derivative value $\left(\frac{d M}{d v}\right)$ and the 2 nd derivative value $\left(\frac{d^{2} M}{d v^{2}}\right)$ for highlight wavelength information. Figure 6 shows the 1st derivative multiplication ratio - voltage characteristics.

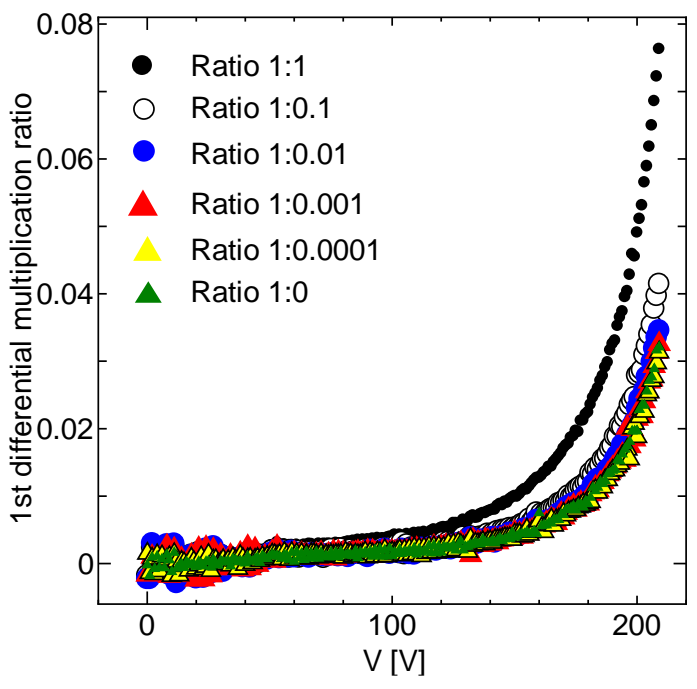

Fig. 6. $\frac{d M}{d t}-$ voltage characteristics.

\section{Classification by multilayer perceptron}

This model is implemented using the PyTorch[6] default set (ver. 1.0.0) and trained with ADAM[7] optimizer using a batch size of 32 and 0.001 learning rate and betas $=(0.9,0.999)$ and default parameter setting. We decrease this learning rate in steps (stepsize400, gamma=0.1). We train the model for 1200 epochs. The model was trained until convergence. We use MLP (Multilayer Perceptron) for 6 type classification. We set three types of MLP model that are $M, d M / d t$, and $d M^{2} / d t^{2}$ type. The MLP has 5 layers that are an input layer and three hidden layers and an output layer. The input nodes number of 
$M, d M / d t$ and $d M^{2} / d t^{2}$ type is 211,210 and 209 respectively.

The first hidden layer, the node number is 150 , set

LeakyReLU (negative_slope $=0.2$ ) activation and Batch

Normalization and dropout rate 0.25 . The second hidden layer, the node number is 100 , set LeakyReLU (negative_slope $=0.2$ ) activation and Batch Normalization and dropout rate 0.25 . The third hidden layer, the node number is 50 , set

LeakyReLU(negative_slope $=0.2$ ) activation and Batch

Normalization and dropout rate 0.25 . The output layer has 6 nodes that are "Ratio(1:0)", "Ratio(1:1)", "Ratio(1:0.1)", "Ratio(1:0.01)", "Ratio(1:0.001)" and "Ratio(1:0.0001)".

Figure 7 shows the MLP image of $M$ type.

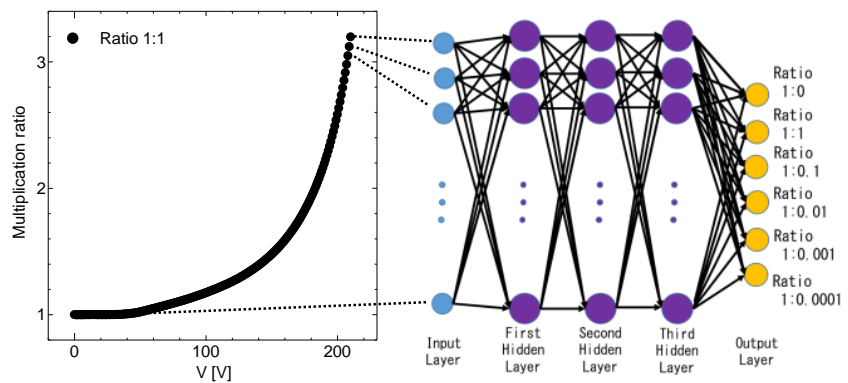

Fig. 7. The MLP image of $M$ type

\section{Classification result}

Table 1 shows the classification result. The classifications of $\operatorname{Ratio}(1: 1)$ and $\operatorname{Ratio(1:0.1)}$ are classified by all preprocessing on this model with a high correct classification rate that is almost 1.0. The classification of Ratio(1:0.01) is classified by preprocessing of $(a)(b)(c)(d)$. The second derivative preprocesses of (e)(f) deteriorates input data for Ratio(1:0.01). Especially, The Ratio(1:0) classification is classified by first derivative preprocess of (d). The dropout process support first derivative process of (d) and second derivative process of (f) for classifying. The best total correct preprocess is (d) that type is first derivative process with dropout.

Table 1. Classification result.

\begin{tabular}{|c|c|c|c|c|c|c|}
\hline $\begin{array}{l}\text { Preprocess } \\
\text { (Dropout) }\end{array}$ & $\begin{array}{c}\text { (a)M } \\
\text { (no dropout) }\end{array}$ & $\begin{array}{c}\begin{array}{c}\text { (b)M } \\
\text { (dropout) }\end{array}\end{array}$ & $\begin{array}{c}\text { (c)dM/dv } \\
\text { (no dropout) }\end{array}$ & $\begin{array}{l}\text { (d)dM/dv } \\
\text { (dropout) }\end{array}$ & \begin{tabular}{|c|} 
(e) $\mathrm{d}^{2} \mathrm{M} / \mathrm{d} \mathrm{v}^{2}$ \\
(no dropout)
\end{tabular} & $\begin{array}{l}\text { (f) } d^{2} M / d v^{2} \\
\text { (dropout) }\end{array}$ \\
\hline $\begin{array}{l}\text { Ratio } \\
(1: 0) \\
\end{array}$ & 0.8083 & 0.0833 & 0.4542 & 1.0000 & 0.8000 & 0.9125 \\
\hline $\begin{array}{l}\text { Ratio } \\
(1: 1)\end{array}$ & 1.0000 & 1.0000 & 1.0000 & 1.0000 & 0.9958 & 1.0000 \\
\hline $\begin{array}{c}\text { Ratio } \\
(1: 0.1)\end{array}$ & 1.0000 & 1.0000 & 1.0000 & 1.0000 & 0.9917 & 1.0000 \\
\hline $\begin{array}{c}\text { Ratio } \\
(1: 0.01)\end{array}$ & 1.0000 & 1.0000 & 0.9958 & 0.9958 & 0.0625 & 0.3250 \\
\hline $\begin{array}{c}\text { Ratio } \\
(1: 0.001)\end{array}$ & 0.0000 & 0.0000 & 0.0000 & 0.0000 & 0.0208 & 0.0000 \\
\hline $\begin{array}{c}\text { Ratio } \\
(1: 0.0001)\end{array}$ & 0.0000 & 0.0000 & 0.0000 & 0.0000 & 0.0125 & 0.0000 \\
\hline Total & 0.6347 & 0.5139 & 0.5750 & 0.6660 & 0.4806 & 0.5396 \\
\hline $\begin{array}{c}\text { Total without } \\
\text { Ratio(1:0.001) } \\
\text { Ratio(1:0.0001) }\end{array}$ & 0.952075 & 0.770825 & 0.8625 & 0.99895 & 0.720825 & 0.809375 \\
\hline
\end{tabular}

The total correct rate is 0.666 . The total correct rate without Ratio (1:0.001) and Ratio (1:0.0001) is 0.99895.

\section{Conclusion}

The preprocess $M$ can classify Ratio(1:1), Ratio(1:0.1), Ratio(1:0.01). The green signal value of Ratio(1:0.01) is almost the same with fluctuation noise. So we achieved our goal on this preprocess. It indicates this method can divide incident lights when there is a high noise. Moreover, the preprocess $d M / d v$ can classify Ratio(1:0). It is indicated the $d M / d v$ preprocess find the green light incident. The current Ratio(1:0) is almost the same Ratio(1:0.001) and Ratio(1:0.0001). The deep learning finds the characteristic by the derivative process. In this report, we can classify the noise level signal (Ratio(1:0.01)) with low multiplication ratio( $M=2$ to 3$)$. We expect more accuracy improvement with high multiplication ratio. It indicated that the deep learning method can improve filter less APD based spectroscope accuracy.

\section{References}

(1) Rajib BasakFan LiuSarfraz QureshiNeelima GuptaCe ZhangRenko de VriesJeroen A. van KanS. Thameem DheenJohan R. C. van der Maarel, "Linearization and Labeling of Single-Stranded DNA for Optical Sequence Analysis", J. Phys. Chem. Lett., vol.10, No.3, pp.316321, 2019.

(2) Sang Mun Bae, Dong-Jun Bae, Eun-Ju Do,Gyungseok Oh, Su Woong Yoo, Gil-Je Lee,Ji Soo Chae, Youngkuk Yun, Sungjee Kim,Ki Hean Kim, Euiheon Chung, Jun Ki Kim,Sung Wook Hwang, Sang Hyoung Park,DongHoon Yang, Byong Duk Ye,Jeong-Sik Byeon, Suk-Kyun Yang,Jinmyoung Joo, Sang-Yeob Kim, and Seung-Jae Myung,"Multi-Spectral FluorescenceImaging of Colon DysplasiaIn VivoUsing a Multi-SpectralEndoscopy System", Translational Oncology, Vol. 12, No. 2,pp. 226-235, 2019.

(3) Yong Joon Choi, Kazuhiro Takahashi, Motoharu Matsuda, Takeshi Hizawa, Yu Moriwaki, Fumihiro Dasai, Yasuyuki Kimura, Ippei Akita, Tatsuya Iwata, Makoto Ishida,"Filter-less fluorescence sensor with high separation ability achieved by the suppression of forward-scattered light in silicon", The Japan Society of Applied Physics Japanese Journal of Applied Physics, Volume 55, Number 4S, pp.4-10, 2016.

(4) Yong Joon Choi ,Kazuhiro Takahashi, Nobuo Misawa, 
Takeshi Hizawa, Tatsuya Iwata, Kazuaki Sawada,

"Multi-wavelength fluorescence detection of submicromolar concentrations using a filter-free fluorescence sensor", Sensors and Actuators B: Chemical, Vol. 256, pp. 38-47, 2018.

(5) Masahiro Akiyama, Kazuya Miyazawa, Kazuaki Sawada, "Highly sensitive filter-less fluorescence detection method using an avalanche photodiode", Journal of Energy and Power Engineering, vol.10(4), pp.268-273, 2017.

(6) Paszke Adam, Gross Sam, Chintala Soumith, Chanan Gregory, Yang Edward, DeVito Zachary, Lin Zeming, Desmaison Alban, Antiga Luca, Lerer Adam, "Automatic differentiation in PyTorch", $31^{\text {st }}$ Conference on Neural Information Processing Systems (NIPS), pp.1-4, 2017.

(7) D.P. Kingma, L.J. Ba, “Adam: A Method for Stochastic Optimization", Published as a conference paper at International Conference on Learning Representations (ICLR), pp.1-15, 2015. 